\title{
Identification and biochemical characterisation of Acanthamoeba castellanii cysteine protease 3
}

Zhixin Wang ${ }^{1 \dagger}$, Duo $\mathrm{Wu}^{1 \dagger}$, Hiroshi Tachibana ${ }^{2}$, Meng Feng ${ }^{1 *}$ and Xun-jia Cheng ${ }^{1 *}$

\begin{abstract}
Background: Acanthamoeba spp. are free-living amoeba that are ubiquitously distributed in the environment. This study examines pathogenic Acanthamoeba cysteine proteases (AcCPs) belonging to the cathepsin L-family and explores the mechanism of ACCP3 interaction with host cells.

Methods: Six ACCP genes were amplified by polymerase chain reaction (PCR). Quantitative real-time PCR was used to analyse the relative mRNA expression of AcCPs during the encystation process and between pre- and post-reactivated trophozoites. To further verify the role of ACCP3 in these processes, AcCP3 recombinant proteins were expressed in Escherichia coli, and the hydrolytic activity of $A c C P 3$ was determined. The influence of the AcCP3 on the hydrolytic activity of trophozoites and the toxicity of trophozoites to human corneal epithelial cells (HCECs) was examined by inhibiting AcCP3 expression using siRNA. Furthermore, the levels of p-Raf and p-Erk were examined in HCECs following coculture with AcCP3 gene knockdown trophozoites by Western blotting.

Results: During encystation, five out of six ACCPs exhibited decreased expression, and only ACCP6 was substantially up-regulated at the mRNA level, indicating that most AcCPs were not directly correlated to encystation. Furthermore, six AcCPs exhibited increased expression level following trophozoite reactivation with HEp-2 cells, particularly AcCP3, indicating that these AcCPs might be virulent factors. After refolding of recombinant AcCP3 protein, the $27 \mathrm{kDa}$ mature protein from the $34 \mathrm{kDa}$ pro-protein hydrolysed host haemoglobin, collagen and albumin and showed high activity in an acidic environment. After AcCP3 knockdown, the hydrolytic activity of trophozoite crude protein against gelatin was decreased, suggesting that these trophozoites had decreased toxicity. Compared with untreated trophozoites or negative control siRNA-treated trophozoites, AcCP3-knockdown trophozoites were less able to penetrate and damage monolayers of HCECs. Western blot analysis showed that the activation levels of the Ras/Raf/Erk/p53 signalling pathways in HCECs decreased after inhibiting the expression of trophozoite ACCP3.
\end{abstract}

Conclusions: AcCP6 was correlated to encystation. Furthermore, ACCP3 was a virulent factor in trophozoites and participated in the activation of the Ras/Raf/Erk/p53 signalling pathways of host cells.

Keywords: Acanthamoeba castellanii, Cysteine protease, Virulence factor, Encystment, p53 pathway

*Correspondence: mengfeng@fudan.edu.cn; xjcheng@shmu.edu.cn †Z Zhixin Wang and Duo Wu contributed equally to this work

1 Department of Medical Microbiology and Parasitology, School of Basic Medical Sciences, Fudan University, Shanghai 200032, China

Full list of author information is available at the end of the article

\begin{abstract}
Background
Acanthamoeba castellanii is a free-living amoeba that can cause Acanthamoeba keratitis in humans after infection as well as granulomatous amoebic encephalitis and cutaneous acanthamoebiasis in immunocompromised individuals $[1,2]$. In recent years, serious Acantham$o e b a$ infections have been associated with an increasing
\end{abstract}

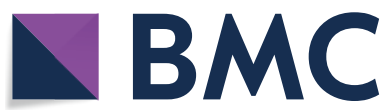

(c) The Author(s) 2020. This article is licensed under a Creative Commons Attribution 4.0 International License, which permits use, sharing, adaptation, distribution and reproduction in any medium or format, as long as you give appropriate credit to the original author(s) and the source, provide a link to the Creative Commons licence, and indicate if changes were made. The images or other third party material in this article are included in the article's Creative Commons licence, unless indicated otherwise in a credit line to the material. If material is not included in the article's Creative Commons licence and your intended use is not permitted by statutory regulation or exceeds the permitted use, you will need to obtain permission directly from the copyright holder. To view a copy of this licence, visit http://creativeco mmons.org/licenses/by/4.0/. The Creative Commons Public Domain Dedication waiver (http://creativecommons.org/publicdomain/ zero/1.0/) applies to the data made available in this article, unless otherwise stated in a credit line to the data. 
number of contact lens wearers and immunocompromised patients. This parasite has a life cycle with two phases, the trophozoite and the cyst, which differ morphologically. When exposed to starvation conditions or a change in osmotic pressure, the trophozoites transform into cysts to better withstand harsh environmental conditions. This parasite readily encysts in response to nutrition source deprivation, osmotic shock or a combination of both [3]. Thus, the growth and encystation of Acanthamoeba trophozoites, which are regulated by a number of biological factors, can lead to persistent infections and influence the pathogenicity of this parasite.

The first stage of Acanthamoeba infection is the interaction between mannose binding protein (MBP) on the surface of the parasite and the mannosylated proteins on the surface of host cells [4]. Next, a variety of proteolytic enzymes are secreted into the host, hydrolysing the host's tissue and resulting in tissue oedema, inflammation and necrosis [5]. Among these enzymes, serine proteases [6] and cysteine proteases (CPs) [7] are the main proteolytic proteins. Studies have shown that many serine proteases with different molecular weights are involved in the degradation of host cells and extracellular matrix [8] during pathogenic Acanthamoeba infection and are inhibited by proteasome inhibitors in a concentration-dependent manner [6].

Many protozoa utilise CPs for nutrient uptake, host infection, tissue invasion and environmental adaptation (encystation). It was well established that pathogenicityrelated CPs, including cysteine protease 1 (CP1), CP2 and $\mathrm{CP} 5$, are expressed during the pathogenic process of Entamoeba histolytica [9]. In Giardia lamblia, CPs and UDP-N-acetylglucosamine pyrophosphorylase are required during encystation. Acanthamoeba expresses many CPs with different molecular weights, including $38.5,43,50,59,70,100$ and $130 \mathrm{kDa}$ proteases [10-13]. After sequencing and analysis, it was found that both the 990 bp A castellanii CP gene (AcCP) [11] and the 1359 bp A. culbertsoni $\mathrm{CP}$ gene (AcCP2) [14] belong to the $\mathrm{L}$ cathepsin $\mathrm{CP}$ family. $A c \mathrm{CP} 2$ contains an Ex3Rx3Wx2N motif in the proregion and a proline/threonine-rich $\mathrm{C}$-terminus. The amino acid sequence of $A c C P$ contains a catalytic site with five residues as well as ERFNIN and GNFD motifs. Moreover, recombinant $A c C P$ protein was capable of hydrolysing host proteins, including haemoglobin, albumin, IgG, IgA and adhesion proteins, suggesting that $A c \mathrm{CP}$ may be an important pathogenic protease in Acanthamoeba [11].

The pathogenic process of Acanthamoeba mainly depends on the hydrolytic activity of the pathogenic proteases secreted by trophozoites, which activate a series of cell signalling pathways in host cells. Several studies have revealed that various downstream molecules are involved, including G-protein-coupled receptors, beta adrenalin receptors [15], Toll-like receptor-4 (TLR4), TLR4-myeloid differentiation primary response gene 88 (MyD88), nuclear factor- $\mathrm{kB}$ (NF-kB), extracellular signalregulated kinase (Erk) [16], phosphatidylinositol-3-kinase (PI3K) [17] and cytosolic phospholipase (A2 $\alpha$ cPLA2 $\alpha$ ) [18]. Due to the complex pathogenic mechanisms of Acanthamoeba infection, cytopathogenic effects in host cells are not solely mediated by these signalling molecules. Instead, changes in the expression levels of other signalling molecules in the host may be involved. The Ras/Raf/Erk signalling pathway is well known as a key intracellular signal transduction pathway regulating cell differentiation, proliferation and apoptosis and can be regulated by various growth factors. When cells are stimulated, Ras protein kinase expression is up-regulated, thereby activating downstream Erk $1 / 2$ protein. Furthermore, the activation of Ras/Raf/ERK signalling can lead to the activation of the tumour suppressor p53. Increased phosphorylation of p53 can up-regulate apoptotic proteins and inhibit bcl-2 proteins, eventually inducing apoptosis [19]. However, whether the Ras/Raf/Erk/ p53 signalling pathways of host cells are involved in the pathogenesis of Acanthamoeba has not yet been verified. In addition, it has not been reported whether the CPs secreted by trophozoites are involved in the activation of the Ras/Raf/Erk/p53 signalling pathways of host cells.

Here, we amplified the CP genes of $A$. castellanii and determined their roles in the pathogenesis and encystation of trophozoites. We confirmed that AcCP3 is specifically required for the pathogenicity and virulence of trophozoites. By potentially activating the Ras/Raf/Erk/ p53 signalling pathway of host cells, AcCP3 may play an important role in the pathogenesis of Acanthamoeba and may be involved in the pathophysiology of Acanthamoeba infection.

\section{Methods \\ Amoeba and cell culture}

A. castellanii (American Type Culture Collection, ATCC 30011), which was originally isolated from a keratitis patient, was cultured axenically in peptone-yeast-glucose (PYG) medium at $26^{\circ} \mathrm{C}$, and trophozoites were harvested in the logarithmic growth phase. Human larynx epidermoid carcinoma cells (HEp-2) purchased from ATCC were cultured in EMEM (Gibco, USA) supplemented with $10 \%$ fetal bovine serum (FBS) (Thermo Fisher Scientific, USA), $100 \mathrm{U} / \mathrm{mL}$ penicillin and $100 \mu \mathrm{g} / \mathrm{mL}$ streptomycin. Human corneal epithelial cells (HCECs) purchased from Bioleaf (Shanghai, China) were grown in DMEM (Gibco, USA) with 10\% FBS, $100 \mathrm{U} / \mathrm{mL}$ penicillin and $100 \mu \mathrm{g} / \mathrm{mL}$ streptomycin. Both cells were cultured in a $37^{\circ} \mathrm{C}$ incubator with $5 \% \mathrm{CO}_{2}$. 


\section{Encystation assays}

Encystation assays were performed as described previously [20] with slight modifications. Briefly, trophozoites from post-logarithmic growth phase cultures were treated with phosphate-buffered saline (PBS; containing $137 \mathrm{mM} \mathrm{NaCl}, 2.7 \mathrm{mM} \mathrm{KCl}, 10 \mathrm{mM} \mathrm{Na} \mathrm{HPO}_{4}, 1.8 \mathrm{mM}$ $\mathrm{KH}_{2} \mathrm{PO}_{4}$ ) with $50 \mathrm{mM} \mathrm{MgCl}$ and $10 \%$ glucose in culture plates at $30{ }^{\circ} \mathrm{C}\left(5 \times 10^{5}\right.$ trophozoites per $\mathrm{mL}$ of medium $)$ for $72 \mathrm{~h}$. The treated materials were collected at $24 \mathrm{~h}$, $48 \mathrm{~h}$ and $72 \mathrm{~h}$ for RNA extraction, respectively.

\section{Transmission electron microscopy}

Transmission electron microscopy was performed to observe the morphology of cysts. The cyst pellets were fixed in $3 \%$ glutaraldehyde and $4 \%$ formaldehyde in phosphate-buffered saline $(0.1 \mathrm{M}, \mathrm{pH} 7.2)$ at $4^{\circ} \mathrm{C}$ for $72 \mathrm{~h}$, washed three times in $0.1 \mathrm{M}$ PBS ( $\mathrm{pH} 7.2$ ), postfixed with $1 \%$ osmium tetroxide in PBS at $4{ }^{\circ} \mathrm{C}$ for $2 \mathrm{~h}$, washed three times in 0.1 M PBS (pH 7.2), dehydrated in an ascending ethanol series and embedded with epoxy resin 618. Ultrathin sections were cut on an LKB Ultratome control unit, stained with $3 \%$ uranyl acetate and lead citrate, and observed under a Tecnai G2 Spirit TWIN transmission electron microscope (FEI, Czech Republic).

\section{Cloning of $A C C P$ genes and expression level analysis}

The CP genes of Acanthamoeba were cloned from the cDNA of trophozoites using the primers listed in the Additional file 1: Table S1 by polymerase chain reaction (PCR). PCR was performed in a 9902 Veriti 96-well Thermal Cycler (Applied Biosystems, USA) $\left(94{ }^{\circ} \mathrm{C}\right.$ for $3 \mathrm{~min}$; 35 cycles of $94{ }^{\circ} \mathrm{C}$ for $15 \mathrm{~s}, 55^{\circ} \mathrm{C}$ for $30 \mathrm{~s}$ and $72{ }^{\circ} \mathrm{C}$ for $1 \mathrm{~min}$; followed by $72{ }^{\circ} \mathrm{C}$ for $7 \mathrm{~min}$ ). The amplified PCR products were purified and ligated into a pMD19-T vector (Takara, Japan), and the nucleotide sequences were obtained by automated sequencing.

A.castellanii mRNA was extracted from trophozoites and cysts using RNeasy ${ }^{\circledR}$ Plus Mini Kit (Qiagen, Hilden, Germany), and cDNA was synthesised using a PrimeScript $^{\circledR}$ 1st strand cDNA synthesis kit (Takara, Japan). Quantitative real-time PCR (qRT-PCR) was carried out in a final reaction volume of $20 \mu \mathrm{L}$ according to the manufacturer's recommendations on an ABI 7500 Real-time PCR system (Applied Biosystems, USA). Reactions were performed in a 96-well plate with TB Green Premix Ex Taq II (Takara, Japan) to analyse the expression level of $A c C P s$. The primers for $A c C P s$ genes and the GAPDH internal reference are listed in Additional file 1: Table S2. The amplification cycling conditions were as follows: $30 \mathrm{~s}$ at $95^{\circ} \mathrm{C}$ and 40 cycles of $5 \mathrm{~s}$ at $95^{\circ} \mathrm{C}$ and $35 \mathrm{~s}$ at $60{ }^{\circ} \mathrm{C}$. Each experiment was performed at least three times.

\section{Expression, purification and refolding of recombinant} AcCP3 protein

The correct plasmids containing $A c \mathrm{CP} 3$ were amplified with primers containing HindIII and $B a m H I$ restriction sites, and the PCR products were ligated into the $\mathrm{pQE}-30$ expression vector (Qiagen, Germany). The sequences of all constructs were confirmed on both strands and analysed with Vector NTI software (Invitrogen, Waltham, USA). Plasmids were transformed into M15 (pREP4) cells (Qiagen, Germany) for protein expression. The selected clones were cultured in Luria-Bertani broth containing $100 \mu \mathrm{g} / \mathrm{mL}$ ampicillin and recombinant AcCP3 (rAcCP3) expression was induced using $1 \mathrm{mM}$ isopropyl- $\beta$-Dthiogalactoside for $3 \mathrm{~h}$ at $37^{\circ} \mathrm{C}$. The recombinant $\mathrm{r} A c \mathrm{CP} 3$ protein was purified using a QIA Express kit in accordance with the manufacturer's instructions. The purity and mass of protein were determined by sodium dodecyl sulphate polyacrylamide gel electrophoresis (SDS-PAGE). Since the N-terminal peptide sequence in recombinant CPs can inhibit the hydrolytic activity of the protease, it was necessary for the recombinant AcCP3 to be refolded to obtain the mature peptide with hydrolytic activity [21]. Refolding of the purified recombinant protein was performed as described previously [11, 21]. In brief, purified $\mathrm{r} A c \mathrm{CP} 3(2 \mathrm{mg})$ was slowly added to $100 \mathrm{~mL}$ refolding buffer, containing $100 \mathrm{mM}$ Tris- $\mathrm{HCl}(\mathrm{pH} 8.0), 1 \mathrm{mM}$ ethylenediaminetetracetic acid (EDTA), $250 \mathrm{mM} \mathrm{L}$-arginine, $5 \mathrm{mM}$ reduced glutathione (GST) and $1 \mathrm{mM}$ oxidised glutathione. The protein was gently stirred at $4^{\circ} \mathrm{C}$ overnight and then dialysed against $10 \mathrm{mM}$ Tris- $\mathrm{HCl}$ (pH 7.5). The obtained $\mathrm{r} A c \mathrm{CP} 3$ was further processed as described previously [22]. In brief, sodium acetate buffers with $\mathrm{pH}$ 4.0 to 7.0 were used to analyse the optimal $\mathrm{pH}$ condition required for obtaining the fully matured $\mathrm{r} A c \mathrm{CP} 3$ enzyme. The concentration of matured $\mathrm{r} A c \mathrm{CP} 3$ enzyme was measured using a protein assay (Bio-Rad, USA).

\section{Analysis of biological characteristics of AcCP3}

To assess the role of AcCP3 in Acanthamoeba pathogenesis, the reactivation of the physiological properties of trophozoites was performed using HEp- 2 cell monolayers as described previously [23, 24]. In brief, HEp-2 cells were cultured in $75 \mathrm{~cm}^{2}$ tissue culture flasks (Corning, USA) at $37{ }^{\circ} \mathrm{C}$ under sterile conditions until the monolayer covered the bottom of the flask completely, at which point the supernatant was removed. Trophozoites $\left(10^{6}\right)$ suspended in $25 \mathrm{~mL}$ physiological $0.9 \% \mathrm{NaCl}$ were inoculated onto the monolayer three times consecutively. Cocultures of amoebae and HEp-2 cells were incubated at $26{ }^{\circ} \mathrm{C}$ until the monolayer was completely lysed. Reactivated trophozoites were collected and total mRNA was extracted. qRT-PCR was performed to analyse the expression level of $A c \mathrm{CP} 3$. In addition, MBP was 
used as a virulence protein in Acanthamoeba [25]. 18S rDNA was used as internal reference [26]. The primers for $A c \mathrm{CP} 3, \mathrm{MBP}$ and $18 \mathrm{~S}$ rDNA are listed in Additional file 1: Table S2.

To determine the effect of AcCP3 on host proteins, haemoglobin (from human blood), collagen (from human placenta) and albumin (from bovine serum) were purchased from Sigma-Aldrich (USA). Each protein (2 mg/ $\mathrm{mL})$ was incubated with matured $\mathrm{rAcCP} 3(100 \mathrm{nM})$ in 50 mM sodium acetate ( $\mathrm{pH} 4.0$ or $\mathrm{pH} 7.0$ ) with 1 mM GST for $3 \mathrm{~h}$ at $37^{\circ} \mathrm{C}$. The reactions were terminated by adding reducing sample buffer and the degradation activity of $\mathrm{r} A c \mathrm{CP} 3$ was analysed by SDS-PAGE.

The protease activities of trophozoite crude proteins were analysed using Novex ${ }^{\mathrm{TM}} 10 \%$ Zymogram Plus (Gelatin, China) Protein Gels (Thermo Fisher Scientific, USA) in accordance with the manufacturer's instructions, and $1.5 \mu \mathrm{g}$ crude protein extract was added to each lane. Various inhibitors for different proteases were used. Crude protein extracts treated and untreated with inhibitors (1 h before electrophoresis) were analysed with zymography. The final concentrations of inhibitors were as follows [27]: for serine proteases, $5 \mathrm{mmol} / \mathrm{L}$ phenylmethylsulphonyl fluoride (PMSF); for metalloproteases, $2 \mathrm{mmol} / \mathrm{L}$ EDTA; for CPs, 40, 60, 80 and $100 \mathrm{mmol} / \mathrm{L}$ N-ethylmaleimide (NEM).

\section{AcCP3 gene silencing}

siRNA targeting the catalytic domain of $A c \mathrm{CP} 3$ was synthesised by RiboBio Co. (China) and based on the cDNA sequence. The sequence of the forward strand was 5'-AGUACAUCAUCAACAACAA-3'. Trophozoites were plated at a density of $5 \times 10^{4}$ cells in 48 -well plates, cultured overnight and then transfected with siRNA $(15 \mu \mathrm{g} /$ $\mathrm{mL}$ ) for $12 \mathrm{~h}$ using SuperFectin ${ }^{\mathrm{TM}}$ In Vitro siRNA Transfection Reagent (Pufei, China). As a control, a negative siRNA provided by RiboBio Co. (China) was also applied to cultured trophozoites. Untreated trophozoites and transfection reagent-treated trophozoites were also processed. After the transfection, the differentially treated trophozoites were harvested to determine the efficacy of the knockdown by examining the expression level of $A c \mathrm{CP} 3$ with qRT-PCR and then for the cytopathic tests.

\section{Effect of ACCP3 knockdown in trophozoites on Acanthamoeba-mediated cytotoxicity}

To determine the effects of reduced $A c \mathrm{CP} 3$ expression on Acanthamoeba-mediated HCECs death, cytotoxicity assays were performed as previously described [28]. Confluent HCEC monolayers in 12-well culture plates (Corning, USA) were incubated with differentially treated trophozoites (ratio $1: 2$ ) at $37{ }^{\circ} \mathrm{C}$ in a $5 \% \mathrm{CO}_{2}$ atmosphere for $24 \mathrm{~h}$. Four different experimental groups were included: group 1, normal cultured confluent HCECs; group 2, HCECs co-cultured with normal trophozoites; group 3, HCECs co-cultured with negative control siRNA transfected trophozoites; group 4, HCECs co-cultured with AcCP3-knockdown trophozoites. The cytopathic effects for the different groups of trophozoites were observed using light microscopy (Olympus, Japan). The trophozoites were detached with cold $2 \mathrm{mM}$ EDTA-PBS buffer, chilled on ice for $20 \mathrm{~min}$ and then harvested. In addition, HCECs were cultured in fresh DMEM medium with CCK- 8 reagent for $2 \mathrm{~h}$, and the cytotoxicity was determined by measuring dehydrogenase release (Cell Counting Kit-8, DOJINDO, Japan). The absorbance of each well was measured at $450 \mathrm{~nm}$ using a Model 680 Microplate Reader (Bio-Rad, USA). To investigate the signalling pathways in HCECs activated by Acanthamoeba, four groups of differentially treated HCECs were harvested after co-culturing with amoeba for $24 \mathrm{~h}$. qRT-PCR was performed to analyse the expression level of the Ras gene (NM_004985.4). The forward strand was 5'-AGGAAGCAAGTAGTAATT GATGGA-3'; the reverse strand was 5'-GCCTGTTTT GTGTCTACTGTTCT-3'. Human GAPDH used as an internal reference, with forward strand 5'-TCACCACCA TGGAGAAGGC-3' and reverse strand 5'-GCTAAG CAGTTGGTGGTGCA-3'

\section{Western blotting assays}

The activation states of Raf, Erk and p53 in differentially treated HCECs were determined using Western blotting assays as previously described [29]. Briefly, four groups of differentially treated HCECs were harvested for Western blotting after coculturing with amoeba for $24 \mathrm{~h}$. The cells were lysed in $100 \mu \mathrm{L}$ lysis buffer $(1 \mathrm{~mL}$ lysis buffer containing $20 \mu \mathrm{L}$ phosphatase inhibitors, $20 \mu \mathrm{L}$ protease inhibitor cocktail, $100 \mu \mathrm{L}$ PBS, $5 \mu \mathrm{L} \mathrm{NP-40} \mathrm{and}$

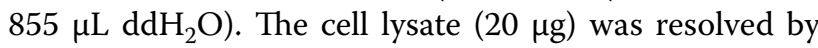
SDS-PAGE and transferred to PVDF membranes (Roche, Switzerland). We used the following primary antibodies purchased from Cell Signaling Technology (USA): rabbit anti-p-Raf (Ser338), rabbit anti-Raf, rabbit antip-Erk (Thr202/Tyr204), rabbit anti-Erk, mouse antip-p53 (Ser15) and mouse anti-p53. HRP-labelled goat anti-mouse and goat anti-rabbit secondary antibodies (Abcam, UK) and rabbit polyclonal to $\beta$-actin (Abcam, UK) were detected with Tanon ${ }^{\mathrm{TM}}$ High-sig ECL Western Blotting Substrate (Tanon, China), observed with an ECL detection system (Tanon, China), and the scanned images were quantified using Image-Pro Plus 4.5.1 software (Media Cybernetics, USA). 


\section{Statistical analysis}

The results of qRT-PCR were calculated using the $2^{-\triangle \triangle C t}$ method. Statistical analyses were performed using GraphPad Prism software (San Diego, CA, USA). Significance was calculated by one-way analysis of variance followed by a Tukey test or Student's $t$-test. Data were expressed as mean $\pm S D$ and at least three independent experiments were performed for each experiment. A $P$ value $<0.05$ was considered significant in all analyses.

\section{Results}

\section{Identification of pathogenic AcCPs}

The sequences encoding full-length $\mathrm{CP}$ genes were amplified using PCR procedures. Four new open reading frames (ORF) corresponding to $\mathrm{CP}$ genes were identified in $A$. castellanii. The sequences of $A c \mathrm{CP} 3$, $A c \mathrm{CP} 8, A c \mathrm{CP} 9$ and $A c \mathrm{CP} 10$ with the accession numbers LC472809, LC472810, LC472812 and LC472813, respectively (Additional file 1: Table S3), have been submitted to the GenBank database. AcCP8 does not present the typical characteristics of CPs. The sequences of AcCP6 (XM004341651) and AcCP7 (XM004358251) from ATCC30011 have also been confirmed in the Neff strain.

The amino acid residues $Q, C, H$ and $N$ that act as the catalytic site of the cathepsin L-family CPs exist in AcCPs. The modified ERFNIN and GNFD motifs that are conserved in cathepsin L-like CP family members have also been observed in $A c C P s$ [30]. We found that while $A c \mathrm{CP} 8$ had no $\mathrm{C}$ active site, the amino acid sequences of the other CPs contained the conserved $\mathrm{C}$ site, which is mainly involved in the refolding of the protease (Additional file 1: Fig. S1). The nucleotide sequence homology of CPs between ATCC30011 and Neff 7 CPs was as high as $99 \%$.

\section{Expression pattern of AcCPs during encystation}

The typical structure of cysts was observed by electron microscopy after $72 \mathrm{~h}$ of encystment (Fig. 1a). qRT-PCR was used to analyse the expression levels of the $A c C P s$ during the encystation process. As shown in Fig. 1b, the mRNA levels of $A c \mathrm{CP} 3, A c \mathrm{CP} 7, A c \mathrm{CP} 8$, $A c \mathrm{CP} 9$ and $A c \mathrm{CP} 10$ were decreased at $24 \mathrm{~h}, 48 \mathrm{~h}$ and $72 \mathrm{~h}$ (with the exception of $A c \mathrm{CP} 7$ at $72 \mathrm{~h}$ ), indicating that these $A c C P s$ may not be involved in encystation. In contrast, $A c \mathrm{CP} 6$ was gradually but substantially upregulated during encystation, suggesting that $A c \mathrm{CP} 6$ may play a uniquely important role in the formation of cysts although most cathepsin L-family CPs were not involved in encystation.
Relationship between AcCPs and protozoan virulence To investigate the role of AcCPs in Acanthamoeba virulence, trophozoites that were axenically grown in vitro for several years were reactivated with HEp-2 cells. The qRT-PCR analysis of $A c C P$ and MBP mRNA expression in pre- and post-reactivated trophozoites is shown in Fig. 2. Compared with pre-reactivated trophozoites, the MBP expression level as well as the expression levels of six of the CPs were up-regulated following trophozoite reactivation, and the expression level of $A c \mathrm{CP} 3$ was significantly increased in trophozoites invading HEp2 cells $(P<0.05$; Fig. 2). These data indicate that $A c C P s$ may play an important role in Acanthamoeba pathogenesis by mediating host cell damage during infection. Furthermore, AcCP3 in particular may act as a potential pathogenic factor during Acanthamoeba invasion.

\section{Refolding of $\mathrm{rAcCP} 3$ and degradation of host proteins}

The AcCP3 gene contains a 993-bp ORF encoding a 329amino acid protein. A portion of the prodomain and the entire mature domain of $A c \mathrm{CP} 3$ was amplified and expressed using a pQE-30 expression vector. AcCP3 was expressed in M15 competent cells as an inclusion body protein with an apparent molecular mass of $34 \mathrm{kDa}$ (Fig. 3a, lane 1). The recombinant protein was purified by Ni-NTA affinity chromatography and then refolded. The refolded sample was further processed under acidreduction conditions to be processed into a fully active enzyme. The size of the fully activated mature $\mathrm{r} A c \mathrm{CP} 3$ was consistent with the predicted size of the mature protease (27 kDa) (Fig. 3a, lane 2). Then, the fully activated $\mathrm{r} A c \mathrm{CP} 3$ was used for an assay of host proteins degradation. SDS-PAGE showed that $\mathrm{r} A c \mathrm{CP} 3$ possesses proteolytic activity against human haemoglobin, collagen and bovine serum albumin. In addition, all these protein substrates were hydrolysed at acidic $\mathrm{pH}$, but not at neutral pH (Fig. 3b-d).

\section{Low proteolytic activity of ACCP3 knockdown trophozoites} To investigate the biological role of AcCP3 in Acanthamoeba trophozoites, trophozoites were transfected with AcCP3-siRNA. qRT-PCR revealed that the mRNA level was significantly reduced in AcCP3 knockdown trophozoites (Fig. 4a). Besides, the expression of other CPs genes was not affected by $A c C P 3$-siRNA (Additional file 1: Fig. S2). There was no significant difference in the growth and proliferation rate of trophozoites between the knockdown and wild-type strains (data not shown). Furthermore, no differences observed in the growth and proliferation rate between superfectin-treated trophozoites (superfectin) and negative control siRNA 


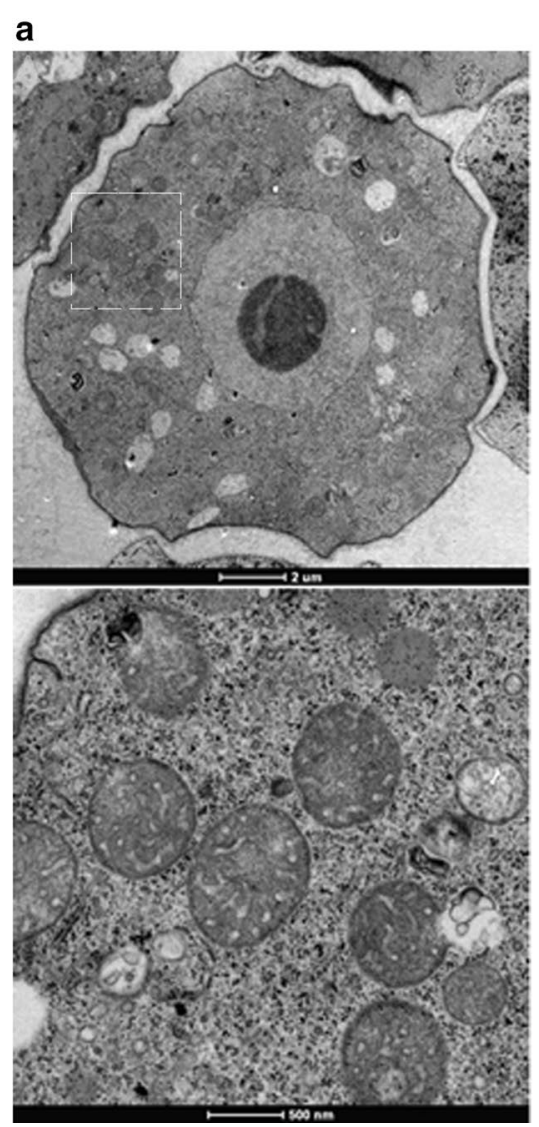

b

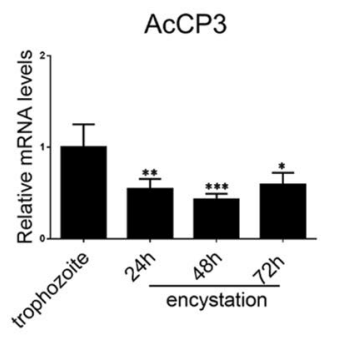

AcCP7

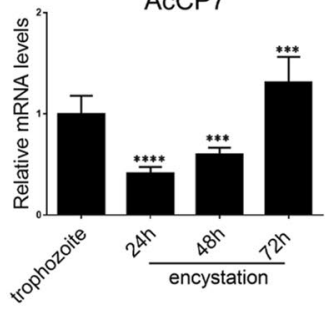

AcCP9

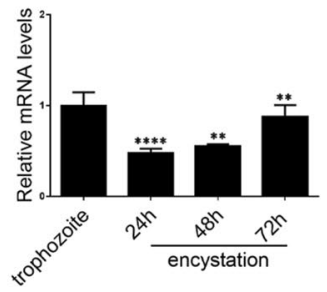

AcCP6

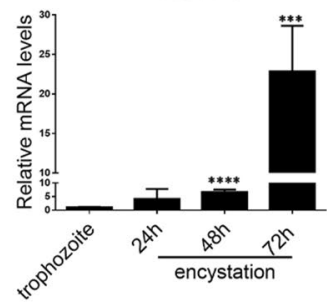

$\mathrm{AcCP8}$

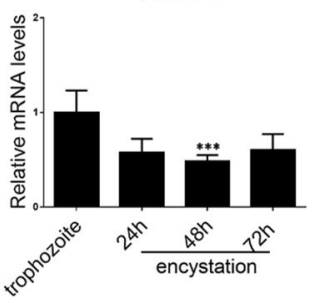

AcCP10

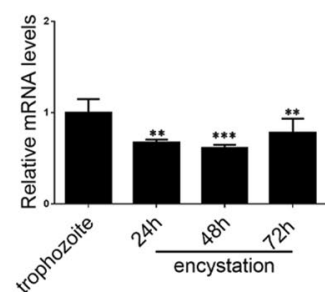

Fig. 1 AcCPs expression levels during encystation using qRT-PCR analysis. a Transmission electron microscopy of cysts. b The experiments were repeated three times, and the average values were presented with vertical bars representing standard deviations. * Means significantly different $(P<0.05)$ by Student's t-test, ${ }^{* *} P<0.01,{ }^{* * *} P<0.001,{ }^{* * * *} P<0.0001$

transfected trophozoites $(\mathrm{Ne})$ compared to untreated trophozoites cultured with PYG. Subsequently, the hydrolytic activity of $A c \mathrm{CP} 3$ knockdown trophozoites was compared to that of wild-type trophozoites and protease inhibitor-treated trophozoites (Fig. 4b, c); $1.5 \mu \mathrm{g}$ of differently treated crude protein extracts was added into lane 1 to lane 8 and lane 10. The crude protein extracts in lane 1 and lane 4, corresponding to wild-type trophozoites, showed high hydrolytic activity. Lane 2 and lane 3 showed that it was not the metalloprotease inhibitor EDTA but the serine proteinase inhibitor PMSF could inhibit this hydrolytic activity. Lanes 5 to 8 showed that NEM could inhibit this hydrolytic activity in a concentration-dependent manner. The ability of PMSF and NEM to inhibit the hydrolytic activity of crude protein extracts from trophozoites confirmed previous results [27]. In lane 9, $1.5 \mu$ g crude protein extract from trophozoites transfected with negative control siRNA showed comparable hydrolytic activity to that from wild-type trophozoites (lane 1 and lane 4), suggesting that the negative control siRNA has little effect on the hydrolytic

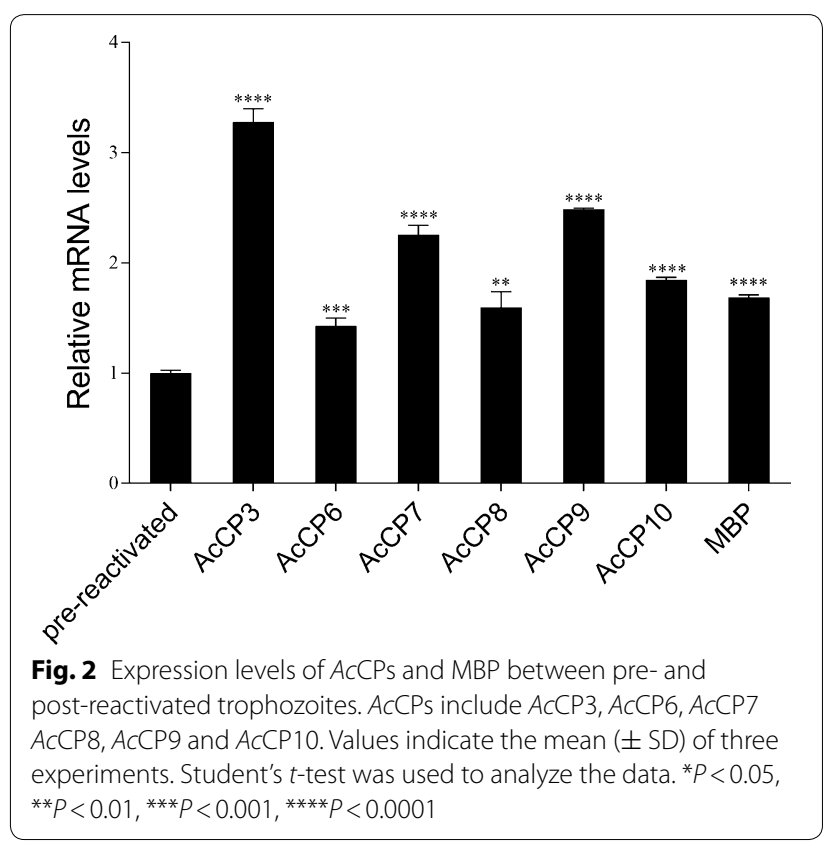


activity. Lane 10 is equally treated as lane 5 . Results in lane 11 showed attenuated hydrolytic activity of trophozoites transfected with AcCP3 siRNA. The zymography above revealed that $A c \mathrm{CP} 3$ was crucial for the proteolytic activity in trophozoites.

\section{Effect of AcCP3 knockdown on protozoa-mediated cytotoxicity}

Cytotoxicity assays were performed with four differently treated HCECs as described in the Methods section. When HCECs were cultured alone, the cells grew into a confluent monolayer and were tightly connected to one another, with a small number of cells lifted off from the dish because of overgrowth (shown as Fig. 5a). The white arrows in Fig. 5b-d indicate the cellular voids formed when HCEC monolayer cells were destroyed by trophozoite penetration. After co-culture of confluent HCECs and wild-type trophozoites, most adherent HCECs became suspended, and the tight connections between the cells were disrupted, resulting in the formation of cellular voids (Fig. 5b), while there were no differences in the formation of cellular voids between negative control siRNA transfected trophozoites and untreated trophozoites (Fig. 5c). In addition, compared with control siRNA transfected trophozoites, trophozoites transfected with AcCP3 siRNA exhibited a decrease in Acanthamoebamediated HCEC cytotoxicity, showing lesser cellular voids and more attached cells (Fig. 5c, d, e). To quantify the cytopathic activity, the dehydrogenase released from cells was detected by CCK-8 kit after co-culture of HCECs and differently treated trophozoites. After 24 $\mathrm{h}$ treatment, HCECs treated with AcCP3 knockdown trophozoites exhibited the highest dehydrogenase release

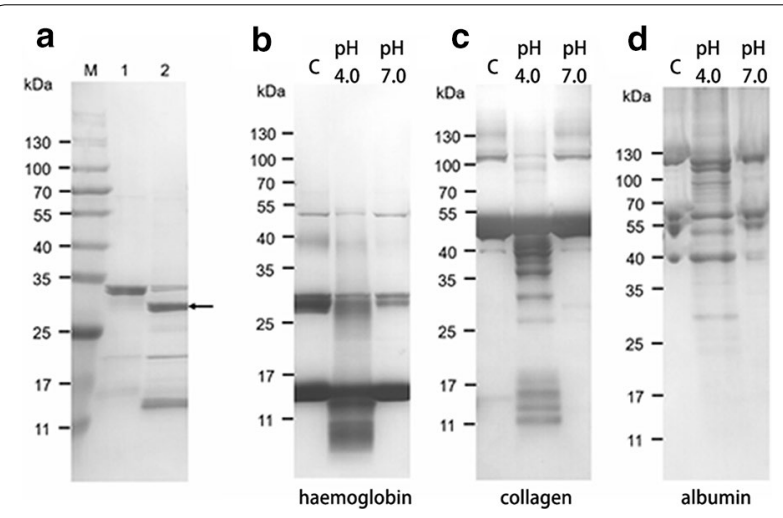

Fig. 3 Expression, purification and refolding of $\mathrm{AACCP} 3$ and degradation of host proteins. a rACCP3 was analyzed by SDS-PAGE. Lane 1, purified rACCP3. Lane 2, refolded rACCP3. b-d Degradation of haemoglobin, collagen and albumin by $\mathrm{r} A C \mathrm{CP} 3$ in a reaction system of pH 4.0 and $\mathrm{pH} 7.0$, respectively. Lane " $\mathrm{C}$ " mean control (without recombinant protein) compared to HCECs treated with normal trophozoites or negative siRNA transfected trophozoites (Fig. 5f). These results indicated that $A c \mathrm{CP} 3$ was an important pathogenic factor for Acanthamoeba to induce cytotoxicity of host cells.

\section{Inhibition of AcCP3 expression antagonized Ras/Raf/Erk signalling in target cells}

Four groups of HCECs treated as described in the Methods section were harvested after co-culturing with trophozoites; qRT-PCR was used to analyse the mRNA expression levels of Ras genes. The data showed (Fig. 6a) that the expression level of K-Ras was significantly increased in HCECs co-cultured with wide-type trophozoites (treat 2). On the contrary, the expression level of K-Ras was significantly decreased in HCECs cocultured with AcCP3 knockdown trophozoites (treat 4) compared with the treat 2 or treat 3 group. This indicated that $A c C P 3$ siRNA could significantly decrease the mRNA expression level of $K$-Ras in Acanthamoebatreated HCECs. In contrast, the mRNA expression levels of $H$-Ras and $N$-Ras showed no differences between the treat 2 and 4 groups (data not shown). Previous studies have demonstrated that Ras recruits Raf kinase into a complex, which mediates Raf phosphorylation. Raf then phosphorylates MEK1 and MEK2, which in turn activate Erk $1 / 2$ by the tandem phosphorylation of threonine and tyrosine residues [31]. Thus, we further examined the levels of p-Raf and p-Erk in HCECs exposed to AcCP3 gene knockdown trophozoites by Western blotting. These data show that untreated trophozoites could significantly increase the phosphorylation levels of Raf, Erk, and p53 proteins in HCECs (Fig. 6, treat 2). Compared to untreated cultured confluent HCECs (treat 1), HCECs co-cultured with untreated trophozoites (treat 2) or HCECs co-cultured with negative control siRNA transfected trophozoites (treat 3), the levels of p-Raf and p-Erk in HCECs co-cultured with AcCP3 siRNA trophozoites (treat 4) were markedly decreased after $24 \mathrm{~h}$ treatment $(P<0.01$; Fig. $6 \mathrm{~b}, \mathrm{c})$. Previous studies have suggested that phosphorylation of p53 might be an important mechanism in Ras/Raf/Erk-induced apoptosis. Thus, we also examined the levels of p-p53 in HCECs cocultured with $A c \mathrm{CP} 3$ knockdown trophozoites. The data showed that the p-p53 was also markedly downregulated after $24 \mathrm{~h}$ treatment $(P<0.01)$. Results above demonstrated that inhibiting the expression of $A c \mathrm{CP} 3$ protein could reduce the Acanthamoeba-mediated phosphorylation levels of Raf, Erk and p53 proteins in HCECs, suggesting that $A c C P 3$ might participate in the activation of Raf, Erk and p53 proteins in HCECs during Acanthamoeba invasion. 

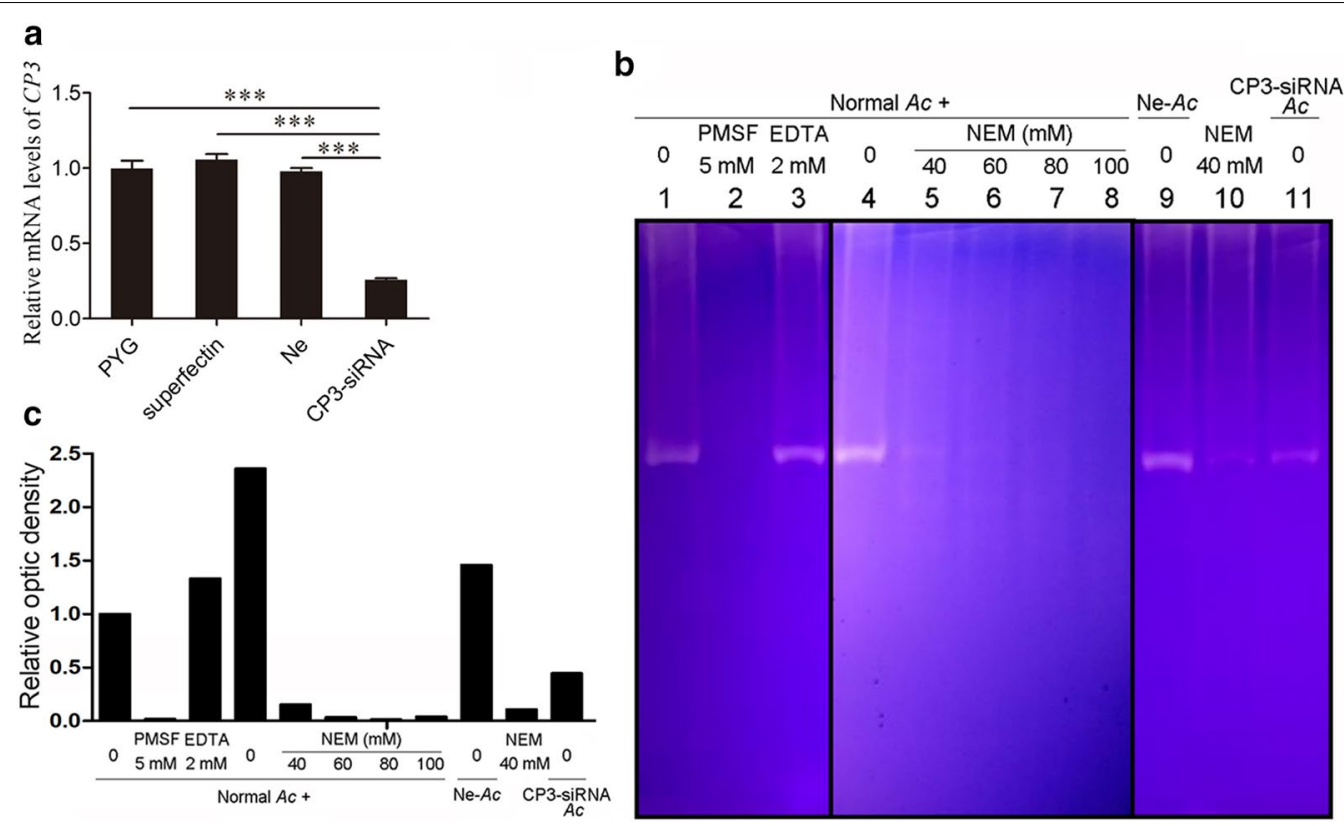

Fig. 4 Zymography showed low hydrolytic activity of AcCP3 gene knockdown trophozoites. a AcCP3 mRNA expression level of wild-type trophozoites (PYG); only superfect-treated trophozoites (superfectin); negative siRNA transfected trophozoites; AcCP3-siRNA transfected trophozoites. b Zymography test of different trophozoite crude extract proteins. Lane 1 and lane 4 showed normal trophozoite crudes proteins with no treatment. Lane 5 and lane 10 showed normal trophozoite crude proteins pretreated with $40 \mathrm{mM}$ NEM. Lane 9 added negative siRNA transfected trophozoite crude proteins. Lane 11 added ACCP3 gene knockdown trophozoite crude proteins. Independent experiments were repeated three times. c The optic densities of the zymography test. Significance was calculated by one-way analysis of variance (ANOVA) followed by a Tukey test. Vertical bars indicate SD. ${ }^{* *} P<0.001$

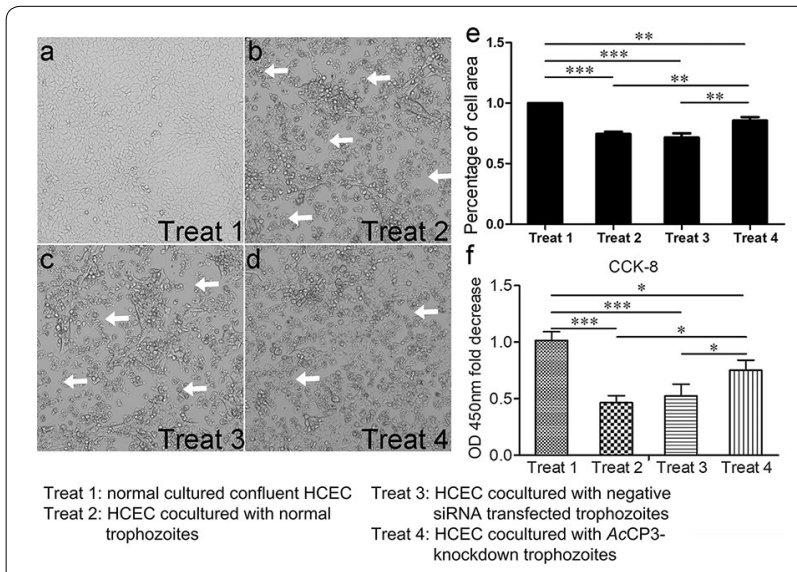

Fig. 5 Microscopy and CCK-8 demonstrating that AcCP3 gene knockdown trophozoites exhibited a decrease in Acanthamoeba-mediated HCEC cytotoxicity. a-d Pictures taken under light microscopy. a Normal cultured confluent HCEC (treat 1). $\mathbf{b}$ HCEC cocultured with normal trophozoites (treat 2). $\mathbf{c}$ HCEC cocultured with negative siRNA transfected trophozoites (treat 3). d HCEC cocultured with ACCP3-knockdown trophozoites (treat 4). e Percentage of cell coverage to the basal area. f OD 450-nm-fold decrease compared to treat $1 \mathrm{HCEC}$ cell. Independent experiments were repeated three times. Vertical bars indicate $S D .{ }^{*} P<0.05$; ${ }^{* *} P<0.01 ;{ }^{* * *} P<0.001$

\section{Discussion}

$\mathrm{CP}$ family proteins are essential to the life cycle of several protozoa by influencing several diverse processes, such as nutrient intake, protein degradation of parasites, immunomodulators, host cell invasion and encystment/ excystment in addition to well-established roles in protein processing and catabolism. They are also an important virulence factor for parasites [32]. It has recently been reported that $A$. castellanii expresses CPs with proteolytic effects [11]. However, little research has been done on the pathogenic and encystment mechanisms of Acanthamoeba CPs. Long-term sterile passage culture of Acanthamoeba trophozoites in vitro may reduce the toxicity of the trophozoites [33]. However, a potential method to reactivate attenuated trophozoite virulence was identified by growing the trophozoites on HEp-2 cell monolayers three times using the Acanthamoeba $1 \mathrm{BU}$ strain (ATCC no. Pra-105) [23, 24]. Using this method, we found that the expression levels of six $A c C$ Ps were higher following reactivation, especially $A c \mathrm{CP} 3$, suggesting that AcCPs may play a pathogenic role in Acanthamoeba. In vitro, AcCP3 could effectively hydrolyse host haemoglobin, collagen and albumin after refolding, and it had strong hydrolytic activity in an acidic environment but almost no hydrolytic activity in a neutral 


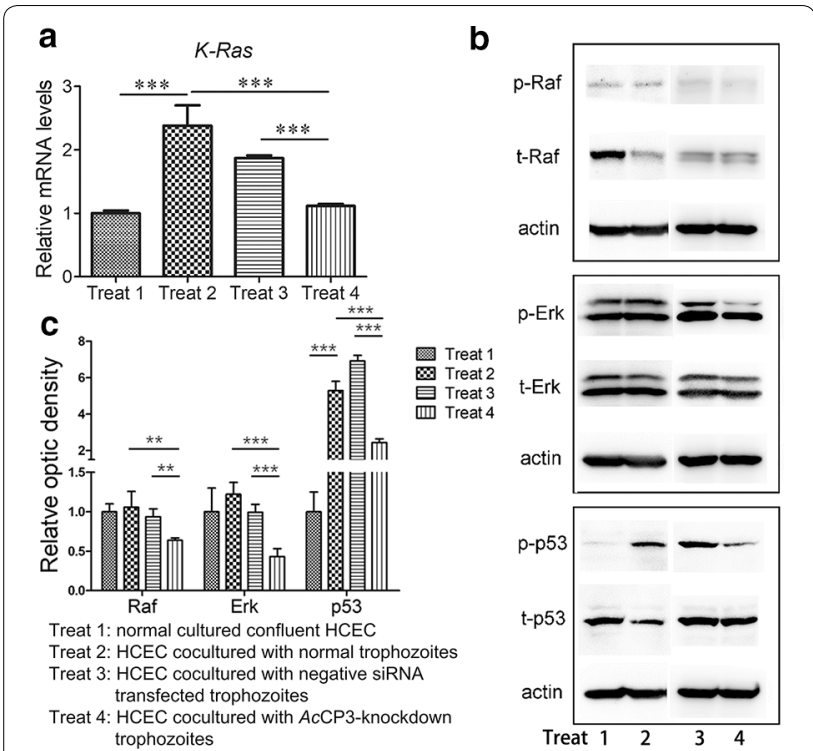

Fig. 6 ACCP3-siRNA significantly decreased the phosphorylation levels of Raf, Erk and p53 in Acanthamoeba-mediated HCEC cells. Four groups of HCEC were treated the same as above. a qRT-PCR analyzed the mRNA expression level of K-Ras gene. $\mathbf{b}$ Western blot analysis of the phosphorylation level of Raf, Erk and p53 proteins in HCEC cells. c The optic densities of phosphorylation levels of Raf, Erk and p53 proteins in HCEC cells. Normal cultured confluent HCEC (treat 1); HCEC cocultured with normal trophozoites (treat 2); HCEC cocultured with negative siRNA transfected trophozoites (treat 3); HCEC cocultured with ACCP3-knockdown trophozoites (treat 4). Independent experiments were repeated three times. Vertical bars indicate SD. ${ }^{*} P<0.05$; ${ }^{* *} P<0.01 ;{ }^{* * *} P<0.001$

environment. This characteristic is similar to that of vivapain from Plasmodium vivax [34] and CsCF-6 from Clonorchis sinensis [21].

The pathogenic process of Acanthamoeba in the host cell is regulated by various signalling molecules. $G$ protein-coupled receptor and $\beta$ adrenalin receptor inhibitors block the activity of trophozoite proteases, thereby affecting the growth, encystation, vitality and pathogenicity of the parasite [15]. The HCECs used in our experiments primarily express the epithelial markers E-cadherin, ZO- 1 and $\beta$-catenin. It has been reported that Toll-like receptor 4 (TLR4) is an important pathogenic target of Acanthamoeba in human corneal epithelial cells (HCECs). After attaching to the surface of host cells, Acanthamoeba activates TLR4, which in turn affects intracellular MyD88, NF-кB and Erk [35]. It has been found that thrombinase and trypsin can activate protease-activated receptor (including PAR1 and PAR2) in HCECs and promote the secretion of pro-inflammatory factors such as IL- 6 , IL- 8 and TNF- $\alpha$, leading to ocular inflammation [36]. Furthermore, studies have shown that Acanthamoeba-induced host cell death is related to PI3K signalling [17]. Acanthamoeba-induced host cell apoptosis is associated with mitochondrial overexpression of pro-apoptotic proteins [37]. It was found that cytosolic phospholipase A2 alpha (cPLA2- $\alpha$ ) is involved in Acanthamoeba-induced apoptosis of HCECs [18]. To determine whether Acanthamoeba AcCP3 interactes with HCECs via the TLR4 receptor, it will be necessary to determine whether CPs can bind to the TLR4 receptor on the surface of HCECs as pathogen-associated molecular patterns. In this study, zymography revealed that the proteolytic activity of trophozoites was decreased following $A c \mathrm{CP} 3$ gene knockdown, and trophozoites after AcCP3 gene silencing exhibited a decrease in Acanthamoebamediated HCEC cytotoxicity. The mechanism of AcCP3 in Acanthamoeba-mediated HCEC cytotoxicity must be confirmed regarding its role in cell signalling pathways.

The Ras/Raf/Erk signalling pathway is the main signalling pathway regulating the occurrence and development of tumours and is important in regulating apoptosis [19]. This pathway activates endogenous apoptotic pathways, such as the release of mitochondrial cytochrome c [38], as well as the activation of caspase-9 [39], caspase-8 [40] and p53 [41]. The ability of the amoeba to activate the Ras/Raf/p53 signalling pathways of host cells during infection has not been verified. Ras signalling molecules include $H$-Ras, $K$-Ras and $N$-Ras [42]. The role of Ras in HCECs has been confirmed, and it has been reported that Ras can regulate the inflammatory response in the cornea of mice [43]. In addition, the Ras/Erk signalling pathway can be activated by hepatocyte growth factor [44] in HCECs. In this study, we found that Acanthamoeba infection mainly affected the expression level of the $K-$ Ras gene in HCECs, and Western blot analysis showed that trophozoites could also increase the phosphorylation levels of Raf, Erk1/2 and p53 in HCECs; taken together, this indicates that Acanthamoeba could activate the Ras/ Raf/ERK/p53 signalling pathways of host cells. Moreover, studies have found that serine and cysteine protease inhibitors can down-regulate Ras pathway-induced apoptosis. Protease inhibitors can inhibit the activation of Ras, which may be related to the involvement of proteases in tumour cell invasion and metastasis [45]. In summary, proteases are involved in the activation of Ras protein and downstream signalling pathways. In this study, the phosphorylation of Ras/Raf/ERK/p53 signalling pathway components in HCECs was decreased when the expression of $A c C P 3$ was inhibited in trophozoites. This suggests that AcCP3 is involved in the activation of the Ras/ Raf/ERK/p53 signalling pathway during the trophozoite pathogenic process.

It is not well understood how CP expression and function change during encystation in Acanthamoeba. We analysed the transcription levels of six genes and found that Acanthamoeba CP AcCP6 was the most highly 
expressed CP gene during the encystation process. This indicates that while most cathepsin L-family CPs are not involved in encystation, $A c \mathrm{CP} 6$ may participate in this process. Of these $\mathrm{CP}$ genes, $A c \mathrm{CP} 6$ emerges as the most highly expressed and exhibits developmental regulation, with expression increasing dramatically during encystation and cyst stages. In general, the process of encystation involves the coordinated secretion of cyst wall materials to the periphery of a cell. It was suggested that trophozoites produce abundant cyst wall proteins, including AcCP6, which are packaged into encystation-specific materials in response to environmental cues. Indeed, cysteine endopeptidases in Giardia were localised to the encystation-specific vesicles during encystation. When fixed cysts were subjected to fluorescence in situ hybridization with $A c \mathrm{CP} 3$ and $A c \mathrm{CP} 6 \mathrm{DNA}$ probes, $A c \mathrm{CP} 3$ and AcCP6 were visualized in both the submembranous cytoplasm and nucleus of cysts (Additional file 1: Fig. S3). Our data indicated that some CPs could also be regulated by acidification of encystation-specific packages; although, CP3 plays a direct role in acidic vesicles, CP6 may act through other pathways and with different mechanisms, which need to be separated from the acid conditions. Both CP3 and CP6 were translated under the membrane, but there was a specific mechanism to regulate the effect of cyst formation-related CP. It had been suggested that encystation vesicles fuse with peripheral vacuoles prior to formation of the cyst wall. The activity of encystationspecific $\mathrm{CP}(\mathrm{AcCP}$ ) toward protein substrates is greatly reduced in an acidic compartment.

\section{Conclusions}

These results suggested that various Acanthamoeba CPs may be required during pathogenic and cyst formation processes. We propose that various CPs, especially $A c \mathrm{CP} 3$ and $A c \mathrm{CP} 6$, play important roles in the regulation of pathogenic and encystment processes. Further study of the pathogenic mechanisms of Acanthamoeba trophozoites will provide a platform for the development of new anti-Acanthamoeba drugs. Further work will be required to clarify the role of the other CPs in Acanthamoeba. The present study preliminarily confirmed that Acanthamoeba trophozoites activate the Ras/Raf/ Erk/p53 signalling pathways of HCECs and contribute to host cell death. The phosphorylation of the Ras/Raf/Erk/ p53 signalling pathway decreased after inhibiting the expression of $A c \mathrm{CP} 3$, indicating that $A c \mathrm{CP} 3$ may be an important pathogenic factor involved in the pathogenesis of trophozoites.

\section{Supplementary information}

Supplementary information accompanies this paper at https://doi. org/10.1186/s13071-020-04474-8.

Additional file 1: Additional figures and tables of identification and biochemical characterisation of Acanthamoeba castellanii cysteine proteinase 3. Figure S1. The catalytic site of cathepsin L-family CPs exist in AcCPs. Structural organization of six A. castellanii cysteine proteases. Numbers indicate the number of amino acid residues forming the predomains, prodomains, or catalytic domains. Figure S2. The AcCPs expression levels of trophozoites after ACCP3 gene silencing using qRT-PCR analysis. PYG: normal cultured trophozoites, Ne: negative siRNA transfected trophozoites, and AcCP3 silencing: AcCP3-knockdown trophozoites. The average values are presented with vertical bars representing standard deviations. Figure S3. Location of ACCP3 and ACCP6 nucleic acids in cyst stage of Acanthamoeba using in situ hybridization. Fixed cysts were subjected to FISH with AcCP3 and AcCP6 DNA probes. AcCP3 (green) and AcCP6 (red) were visualized in both submembranous cytoplasm and nucleus of cysts (a and b). DAPI-stained cell nuclei (blue) are shown for orientation. Table S1. Primers used for amplification of A.castellanii genes. Table S2. Primers used for qRT-PCR of A.castellanii genes. Table S3. The information of AcCPs.

\section{Abbreviations}

AcCPs: Acanthamoeba cysteine proteases; HCECs: Human corneal epithelial cells; CP: Cysteine proteases; TLR4: Toll-like receptor-4; MyD88: TLR4-Myeloid differentiation primary response gene; NF-kB: Nuclear factor-kB; Erk: Extracellular signal-regulated kinase; PI3K: Phosphatidylinositol-3-kinase; PBS: Phosphate-buffered saline; qRT-PCR: Quantitative real-time polymerase chain reaction; SDS-PAGE: Sodium dodecyl sulphate polyacrylamide gel electrophoresis; EDTA: Ethylenediaminetetracetic acid; GST: Glutathione; PMSF: Phenylmethylsulphonyl fluoride; ORF: Open reading frames.

\section{Acknowledgements}

Not applicable.

\section{Authors' contributions}

XC and MF designed the study. ZW, DW, MF conducted the work. XC acquired funding. ZW and DW wrote the first draft of the manuscript. XC, MF and HT revised the manuscript. All authors read and approved the final manuscript.

\section{Funding}

This research was funded by the National Natural Science Foundation of China. (NSFC, 81572020).

Availability of data and materials

All data generated or analyzed during this study are included in the article.

Ethics approval and consent to participate

Not applicable.

Consent for publication

Not applicable.

Competing interests

The authors declare that they have no competing interests.

\section{Author details}

1 Department of Medical Microbiology and Parasitology, School of Basic Medical Sciences, Fudan University, Shanghai 200032, China. ${ }^{2}$ Department of Infectious Diseases, Tokai University School of Medicine, Isehara, Kanagawa 259-1193, Japan.

Received: 12 August 2020 Accepted: 5 November 2020

Published online: 23 November 2020 


\section{References:}

1. Wu D, Feng M, Wang ZX, Qiao K, Tachibana H, Cheng XJ. Molecular and biochemical characterization of key enzymes in the cysteine and serine metabolic pathways of Acanthamoeba castellanii. Parasit Vectors. 2018;11:604.

2. Khan NA. Acanthamoeba: biology and increasing importance in human health. FEMS Microbiol Rev. 2006;30:564-95.

3. Anwar A, Khan NA, Siddiqui R. Combating Acanthamoeba spp. cysts: what are the options? Parasit Vectors. 2018;11:26.

4. Garate M, Cao Z, Bateman E, Panjwani N. Cloning and characterization of a novel mannose-binding protein of Acanthamoeba. J Biol Chem. 2004;279:29849-56.

5. Ng SL, Nordin A, Abd Ghafar N, Suboh Y, Ab Rahim N, Chua KH. Acanthamoeba-mediated cytopathic effect correlates with MBP and AhLBP mRNA expression. Parasit Vectors. 2017;10:625.

6. Kim WT, Kong HH, Ha YR, Hong YC, Jeong HJ, Yu HS, et al. Comparison of specific activity and cytopathic effects of purified $33 \mathrm{kDa}$ serine proteinase from Acanthamoeba strains with different degree of virulence. Korean J Parasitol. 2006:44:321-30.

7. Moon EK, Hong Y, Chung DI, Kong HH. Cysteine protease involving in autophagosomal degradation of mitochondria during encystation of Acanthamoeba. Mol Biochem Parasitol. 2012;185:121-6.

8. Khan NA, Jarroll EL, Panjwani N, Cao Z, Paget TA. Proteases as markers for differentiation of pathogenic and nonpathogenic species of Acanthamoeba. J Clin Microbiol. 2000;38:2858-61.

9. Betanzos A, Bañuelos C, Orozco E. Host invasion by pathogenic amoebae: Epithelial disruption by parasite proteins. Genes. 2019;10:618.

10. Ramírez-Rico G, Martínez-Castillo M, de la Garza M, Shibayama M, Serrano-Luna J. Acanthamoeba castellanii proteases are capable of degrading iron-binding proteins as a possible mechanism of pathogenicity. J Eukaryot Microbiol. 2015;62:614-22.

11. Hong Y, Kang JM, Joo SY, Song SM, Lê HG, Thái TL, et al. Molecular and biochemical properties of a cysteine protease of Acanthamoeba castellanii. Korean J Parasitol. 2018;56:409-18.

12. Hong YC, Hwang MY, Yun HC, Yu HS, Kong HH, Yong TS, et al. Isolation and characterization of a cDNA encoding a mammalian cathepsin L-like cysteine proteinase from Acanthamoeba healyi. Korean J Parasitol. 2002:40:17-24.

13. Alfieri SC, Correia CE, Motegi SA, Pral EM. Proteinase activities in total extracts and in medium conditioned by Acanthamoeba polyphaga trophozoites. J Parasitol. 2000;86:220-7.

14. Yun HC, Kim KY, Park SY, Park SK, Park H, Hwang UW, et al. Cloning of a cysteine proteinase gene from Acanthamoeba culbertsoni. Mol Cells. 1999;9:491-6.

15. Aqeel $Y$, Siddiqui $R$, Manan Z, Khan NA. The role of $G$ protein coupled receptor-mediated signaling in the biological properties of Acanthamoeba castellanii of the T4 genotype. Microb Pathog. 2015;81:22-7.

16. Ren MY, Wu XY. Toll-like receptor 4 signalling pathway activation in a rat model of Acanthamoeba Keratitis. Parasite Immunol. 2011;33:25-33.

17. Sissons J, Kim KS, Stins M, Jayasekera S, Alsam S, Khan NA. Acanthamoeba castellanii induces host cell death via a phosphatidylinositol 3-kinasedependent mechanism. Infect Immun. 2005:73:2704-8.

18. Tripathi T, Abdi M, Alizadeh H. Role of phospholipase A(2) (PLA(2)) inhibitors in attenuating apoptosis of the corneal epithelial cells and mitigation of Acanthamoeba keratitis. Exp Eye Res. 2013;113:182-91.

19. Cagnol S, Chambard JC. ERK and cell death: mechanisms of ERK-induced cell death-apoptosis, autophagy and senescence. FEBS J. 2010;277:2-21.

20. Aqeel $Y$, Siddiqui $R$, Iftikhar $H$, Khan NA. The effect of different environmental conditions on the encystation of Acanthamoeba castellanii belonging to the T4 genotype. Exp Parasitol. 2013;135:30-5.

21. Na BK, Kang JM, Sohn WM. CsCF-6, a novel cathepsin F-like cysteine protease for nutrient uptake of Clonorchis sinensis. Int J Parasitol. 2008:38:493-502.

22. Na BK, Bae YA, Zo YG, Choe Y, Kim SH, Desai PV, et al. Biochemical properties of a novel cysteine protease of Plasmodium vivax, vivapain-4. PLoS Negl Trop Dis. 2010;4:e849.

23. Koehsler M, Leitsch D, Duchene M, Nagl M, Walochnik J. Acanthamoeba castellanii: growth on human cell layers reactivates attenuated properties after prolonged axenic culture. FEMS Microbiol Lett. 2009;299:121-7.

24. Coulon C, Dechamps N, Meylheuc T, Collignon A, McDonnell G, Thomas $V$. The effect of in vitro growth conditions on the resistance of Acanthamoeba cysts. J Eukaryot Microbiol. 2012;59:198-205.
25. Kim JH, Matin A, Shin HJ, Park H, Yoo KT, Yuan XZ, et al. Functional roles of mannose-binding protein in the adhesion, cytotoxicity and phagocytosis of Acanthamoeba castellanii. Exp Parasitol. 2012;132:287-92.

26. Wu D, Qiao K, Feng M, Fu Y, Cai J, Deng Y, et al. Apoptosis of Acanthamoeba castellanii trophozoites induced by oleic acid. J Eukaryot Microbiol. 2018;65:191-9.

27. Serrano-Luna JJ, Cervantes-Sandoval I, Calderon J, Navarro-Garcia F, Tsutsumi V, Shibayama M. Protease activities of Acanthamoeba polyphaga and Acanthamoeba castellanii. Can J Microbiol. 2006:52:16-23.

28. Alsam S, Kim KS, Stins M, Rivas AO, Sissons J, Khan NA. Acanthamoeba interactions with human brain microvascular endothelial cells. Microb Pathog. 2003;35:235-41

29. Feng D, Wang B, Ma Y, Shi W, Tao K, Zeng W, et al. The Ras/Raf/Erk pathway mediates the subarachnoid hemorrhage-induced apoptosis of hippocampal neurons through phosphorylation of p53. Mol Neurobiol. 2016;53:5737-48.

30. Bruchhaus I, Loftus BJ, Hall N, Tannich E. The intestinal protozoan parasite Entamoeba histolytica contains 20 cysteine protease genes, of which only a small subset is expressed during in vitro cultivation. Eukaryot Cell. 2003;2:501-9.

31. McCubrey JA, Steelman LS, Chappell WH, Abrams SL, Wong EW, Chang $F$, et al. Roles of the Raf/MEK/ERK pathway in cell growth malignant transformation and drug resistance. Biochim Biophys Acta. 2007;1773:1263-84

32. Sajid M, Mckerrow JH. Cysteine proteases of parasitic organisms. Mol Biochem Parasitol. 2002;120:1-21.

33. Mazur T, Hadas E. The effect of the passages of Acanthamoeba strains through mice tissues on their virulence and its biochemical markers. Parasitol Res. 1994;80:431-4.

34. Na BK, Shenai BR, Sijwali PS, Choe Y, Pandey KC, Singh A, et al. Identification and biochemical characterization of vivapains, cysteine proteases of the malaria parasite Plasmodium vivax. Biochem J. 2004;378:529-38.

35. Ren M, Gao L, Wu X. TLR4: the receptor bridging Acanthamoeba challenge and intracellular inflammatory responses in human corneal cell lines. Immunol Cell Biol. 2010;88:529-36.

36. Tripathi T, Abdi M, Alizadeh H. Protease-activated receptor 2 (PAR2) is upregulated by Acanthamoeba plasminogen activator (aPA) and induces proinflammatory cytokine in human corneal epithelial cells. Invest Ophthalmol Vis Sci. 2014:55:3912-21.

37. Chusattayanond AD, Boonsilp S, Kasisit J, Boonmee A, Warit S. Thai Acanthamoeba isolate (T4) induced apoptotic death in neuroblastoma cells via the Bax-mediated pathway. Parasitol Int. 2010;59:512-6.

38. Zhang X, Shan P, Sasidhar M, Chupp GL, Flavell RA, Choi AMK, et al. Reactive oxygen species and extracellular signal-regulated kinase 1/2 mitogen-activated protein kinase mediate hyperoxia-induced cell death in lung epithelium. Am J Respir Cell Mol Biol. 2003;28:305-15.

39. Glotin AL, Calipel A, Brossas JY, Faussat AM, Treton J, Mascarelli F. Sustained versus transient ERK1/2 signaling underlies the anti- and proapoptotic effects of oxidative stress in human RPE cells. Invest Ophthalmol Vis Sci. 2006:47:4614-23.

40. Yang R, Piperdi S, Gorlick R. Activation of the RAF/mitogen-activated protein/extracellular signal-regulated kinase kinase/extracellular signalregulated kinase pathway mediates apoptosis induced by chelerythrine in osteosarcoma. Clin Cancer Res. 2008;14:6396-404.

41. Wu Z, Wu LJ, Tashiro S, Onodera S, Ikejima T. Phosphorylated extracellular signal-regulated kinase up-regulated p53 expression in shikonin-induced HeLa cell apoptosis. Chin Med J. 2005;118:671-7.

42. Cox AD, Der CJ. Ras history: The saga continues. Small GTPases. 2010;1:2-27.

43. Sonoda K, Sakamoto T, Yoshikawa H, Ashizuka S, Ohshima Y, Kishihara K, et al. Inhibition of corneal inflammation by the topical use of Ras farnesyltransferase inhibitors: selective inhibition of macrophage localization. Invest Ophthalmol Vis Sci. 1998;39:2245-51.

44. Liang Q, Mohan RR, Chen L, Wilson SE. Signaling by HGF and KGF in corneal epithelial cells: Ras/MAP kinase and Jak-STAT pathways. Invest Ophthalmol Vis Sci. 1998:39:1329-38.

45. Troll W, Frenkel K, Wiesner R. Protease inhibitors as anticarcinogens. J Natl Cancer Inst. 1984;73:1245-50.

\section{Publisher's Note}

Springer Nature remains neutral with regard to jurisdictional claims in published maps and institutional affiliations. 